\title{
NOVA en la definición de políticas públicas en Latinoamérica
}

\author{
NOVA in the definition of public policies in \\ Latin America
}

\author{
NOVA na definição de novas políticas públicas \\ na América Latina
}

doi: 10.1590/0102-311X00312620

João Peres 1

Simón Barquera 2

Ximena Moratorio 3

10 Joio e o Trigo, São Paulo, Brasil.

${ }^{2}$ Centro de Investigación en Nutrición

y Salud, Instituto Nacional de Salud

Pública, Cuernavaca, México.

3 Ministerio de Salud, Montevideo,

Uruguay.

Correspondencia

J. Peres

O Joio e o Trigo.

Rua Rocha 238, São Paulo, SP

01330-000, Brasil.

joaomdperes@gmail.com
El rápido crecimiento de la prevalencia de exceso de peso y obesidad, así como de enfermedades crónicas no transmisibles, desafía a los gobiernos y a las sociedades de toda Latinoamérica. La región ofrece como respuesta una serie de políticas públicas y de teorías para intentar enfrentarse a este problema 1. Comenzando por la clasificación NOVA 2, en la década pasada, seguida por la creación de impuestos especiales sobre bebidas azucaradas, restricciones a la oferta de ultraprocesados en el entorno escolar, formulación de guías alimentarias que recomiendan expresamente evitar el consumo de ultraprocesados, así como por la adopción de sistemas de etiquetado frontal, con el fin de desincentivar la ingestión de esos productos.

Conversamos con dos personas directamente implicadas en la formulación de recomendaciones y políticas públicas para analizar cómo se implementó NOVA en la agenda adoptada en diferentes partes de Latinoamérica. Simón Barquera es director del Área de Investigación en Políticas y Programas de Nutrición del Instituto Nacional de Salud Pública de México. Como coinvestigador de la Encuesta Nacional de Nutrición y Salud de México, él intentaba, al mismo tiempo que los colegas brasileños implicados en la creación de la clasificación NOVA, entender por qué los índices de enfermedades crónicas, en especial de diabetes, crecían entre los mexicanos 3 . Barquera fue un participante directo en la formulación de las evidencias científicas que fundamentaron dos políticas públicas que intentan desestimular el consumo de ultraprocesados. Primero, el país creó un impuesto especial sobre bebidas azucaradas 4 . Más recientemente, adoptó un sistema de etiquetado frontal con un formato de alertas, inspirado en el modelo creado en Chile 5. El perfil de nutrientes implementado en México, para definir qué productos deben estampar sellos negros con forma de octógono, es el de la Organización Panamericana de la Salud (OPS) 6. Este sistema se definió en una clara sintonía con NOVA. 
Ximena Moratorio, ex-coordinadora del Programa de Nutrición del Ministerio de Salud de Uruguay, participó directamente en la elaboración de la Guía Alimentaria para la Población Uruguaya 7. En esta última, aunque también es la regla de oro de las directrices brasileñas, aparece uno de los mensajes-clave de la versión uruguaya: prefiera siempre alimentos in natura o mínimamente procesados, así como preparaciones culinarias a alimentos ultraprocesados. En base a la guía, el gobierno promulgó una serie de políticas públicas que tiene como objetivo cumplir con el propósito de la guía. En 2018, el país adoptó un sistema de etiquetado frontal con un formato de alertas, todavía en fase de implementación. En medio de la pandemia de COVID-19, el Ministerio de Desarrollo Social ejecutó la orientación oficial para limitar la donación de ultraprocesados por parte de corporaciones de alimentación 8 . 


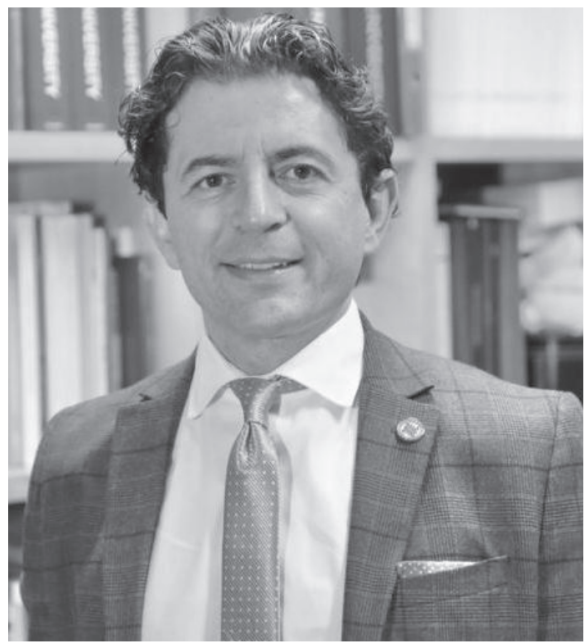

Dr. Simón Barquera (foto: archivo personal).

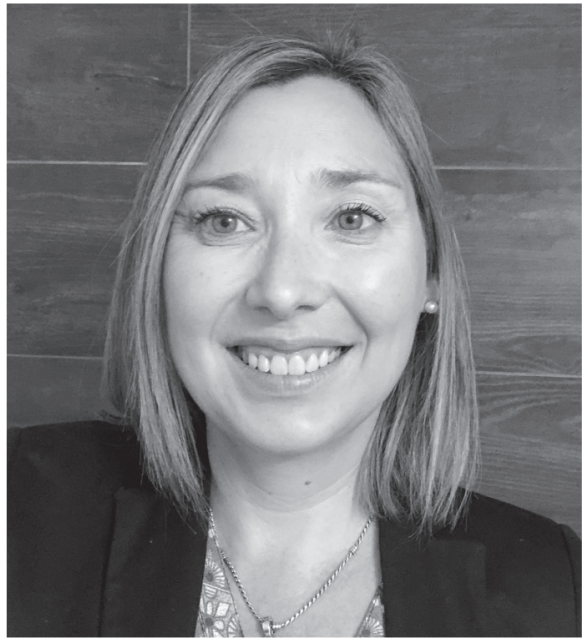

Dra. Ximena Moratorio (foto: archivo personal).

João ¿Cómo ven los puntos fuertes y débiles de la clasificación NOVA?, ¿cómo evalúan la contribución que da a la investigación científica? Y ¿qué es lo que todavía ven como puntos que se deben mejorar en la clasificación?

Ximena Para nosotros fue -en términos de Uruguay- una clasificación que fue adoptada lentamente, por supuesto. Personalmente, como nutricionista formada desde otra lógica para analizar la alimentación, yo creo que tuvimos que cambiar con la aparición de esta clasificación el paradigma de la alimentación. Yo, cuando me formé, pensando en guías de consumo, calculando los nutrientes, y lo que más importaba era que nos cerrara perfecto: el calcio, el hierro, las vitaminas. Esto cambia totalmente el foco de la alimentación y pone el foco, no sólo en los nutrientes, sino en las comidas, en la sostenibilidad de los alimentos, en cómo se producen, se consumen, se comercializan. Entonces, me parece que es una mirada mucho más amplia, más completa. Creo también que la clasificación NOVA le puso un nombre a esos alimentos, que a veces no sabíamos cómo nombrar. Yo siempre hago la comparación, cuando estoy dando una charla sobre la guía alimentaria, que me ha tocado realizar varias veces, sobre cómo cambió, por ejemplo, nuestro ícono; antes, uno dividía los grupos de alimentos en cereales, en dulces... y no importaba mucho qué era lo que estaba allá dentro. Hoy por hoy, tenemos como otra mirada, donde se ven otros componentes de los alimentos, que no solamente tienen que ver con cuánto azúcar aportan, sino cuáles realmente son ingredientes, y cuántos son de alguna forma extractos o subproductos de ingredientes y de alimentos.

Y, específicamente, creo que la principal contribución es eso: cambió el paradigma de la alimentación con una mirada mucho más completa del tema. Creo que nuclea diferentes miradas que tienen que ver con la sostenibilidad del ambiente, con los procesos de comercialización, de industrialización, y no solo los nutricionistas, mirando los nutrientes.

Desde el punto de vista de las debilidades, ¿̨cuáles han sido nuestras dificultades a la hora de adoptar esta clasificación? Nosotros incluimos en la guía uno de nuestros mensajes que, específicamente, habla de evitar en el día a día el consumo de ultraprocesados con excesiva cantidad de grasas, azúcar y sal. Y tuvimos, por supuesto, que enfrentar muchos obstáculos para colocar esa clasificación. Nosotros comenzamos el proceso de discusión de la guía en el año 2015, cuando era todavía relativamente nueva esta clasificación, cuando no había sido de alguna forma adoptada a nivel más internacional y, por supuesto, que hubo muchos detractores, fundamentalmente detractores vinculados a la industria, 
donde, específicamente, les puedo contar que han enviado hasta en su momento una nota dirigida al Ministro de Salud para solicitar que no se incluyera el término ultraprocesados en nuestra guía.

Entonces, me parece que un punto débil en la clasificación es que no hay como una coincidencia en lo que son las clasificaciones, desde el punto de vista químico o incluso regulatorio, porque si uno busca el término ultraprocesados, por ejemplo, en nuestro reglamento bromatológico no está, y el procesamiento de alimentos, desde la perspectiva de quienes trabajan en lo que es la producción de alimentos a nivel industrial, se entiende con otros términos. Me parece que hay que avanzar un poco más en lo que tiene que ver con la clasificación en términos regulatorios, en la nomenclatura, y también buscar otras nomenclaturas que puedan tener las mismas coincidencias con lo que son las clasificaciones más químicas. Porque los químicos y los que están relacionados con la industria de la alimentación son generalmente nuestros principales detractores, porque aducen que se les está colocando una connotación negativa al procesamiento industrial, que muchas veces, por supuesto, hace que los alimentos puedan ser más inocuos u ofrecer algunas ventajas sensoriales.

Simón Nosotros trabajamos en un centro de investigación que asesora al gobierno. Es un think tank, más que un grupo de implementación de políticas alimentarias. Entonces, lo que a nosotros nos pasó es que llevábamos años en las encuestas nacionales de nutrición y salud, recolectando datos de dieta y, pues, batallando mucho con ese viejo paradigma, donde uno hace un cuestionario, un recordatorio de qué comió todo el día anterior la persona, y luego eso se convierte a preparación, y las preparaciones se convierten a nutrientes y micronutrientes y, luego de ahí, se trata de hacer alguna inferencia sobre las dietas. Eso tiene una cantidad de retos y una cantidad de error gigantesco, no solo por el método de recolectar la información, sino después cómo esa información se convierte a micronutrientes, pero, bueno, ese sería uno de los métodos tradicionales que estaba de moda, yo diría que usual desde hace 30 años, ¿no? Conforme fuimos avanzando un poco en tratar de identificar determinantes de obesidad, pues vimos, entre algunas de las cosas que nos interesaba, esta moda de analizar más patrones de dieta que nutrimentos. Y, bueno, algunos de los estudios que empezamos a hacer para entender patrones de dieta separaban alimentos procesados o no procesados, y nos empezamos a interesar mucho por esa forma de ver las cosas. Cuando salió la clasificación NOVA, creo que estábamos esperando algo así. Creo que realmente es una forma sencilla de poder clasificar y analizar un poco la relación de lo que consumen las poblaciones, con sus patrones de salud o de enfermedad y, bueno, en los primeros intentos que hicimos por utilizar este sistema, pues rápidamente encontramos que permite identificar asociaciones muy claras entre ciertas formas de consumir alimentos, y ciertos patrones sobre todo de enfermedades crónicas. Creo que otra cosa que ayudó mucho fue entender la forma de identificar políticas que permitan mejorar la alimentación, y ahí ese perfil que adopta OPS, donde lo que se evalúa es el alimento de forma integral. Entonces, pues como que, de alguna manera, quedan poco favorecidos los alimentos ultraprocesados por tener altas cantidades, proporcional a lo que son, de ingredientes críticos. Esto también es algo que nos parece muy acertado, ahora que viene la preocupación por mejorar las dietas, implementando estrategias como el etiquetado de advertencia o impuestos.

Por ello, este perfil resulta ser muy útil y México lo adopta, además tuvimos la suerte de que hasta ahora todo el proceso ha favorecido esta postura. Nosotros participamos en el diseño aquí en México, y ya quedó autorizada a nivel de ley, y a nivel de norma, aunque todavía no está implementada. Nos parece que es una clasificación sumamente práctica, y como mencionaba Ximena, tiene mucha oportunidad de mejora. Nosotros vemos problemas, por ejemplo, dependiendo de las decisiones que uno toma para dónde quedan algunos productos. Por ejemplo, aquí en México, la tortilla es un alimento que es ampliamente consumido, pero la forma en la que se hace varía mucho. Hay tortillerías que serían el equivalente a panaderías en cada esquina, en todo México. Algunas utilizan unas harinas que son industrializadas que tienen hasta celulosa, que les retiran la fibra del maíz, y le añaden celulosa para mantener la humedad o les añaden colores; otras tortillerías usan un poco de harina procesada de maíz, procesada a nivel local. Entonces, siendo esa la base de la dieta, clasificar en una gran encuesta la tortilla, como procesada, ultraprocesada, o como no procesada, pues no nos ayuda mucho, porque la forma de preparación varía mucho.

João Quisiera hablar un poco más de este tema, de las críticas contra la NOVA, primero hablando desde el punto de vista la industria, ¿cómo evalúan ustedes la oposición que hace la industria especialmente de este término ultraprocesados? 
Ximena Nosotros vemos una oposición fundamentalmente de la industria como tal, porque, por supuesto, se ven atacados en productos en los cuales existe crecimiento del consumo por parte de la población, en particular de Uruguay, pero entiendo que en toda la región es muy grande. Entonces, de alguna forma, sienten esto como una amenaza. Claramente, en nuestro país, por ejemplo, en el estudio de la OPS, que analizó un periodo hasta el año 2000, Uruguay presentó la tendencia más ascendente en el consumo de ultraprocesados, comparado con los otros países de la región 9. Prácticamente se duplicaron los consumos en el periodo de estudio, con un creciente consumo sobre todo de bebidas azucaradas, pero también de otros tipos de productos ultraprocesados, llegando a más de $140 \mathrm{~kg}$ anuales. Realmente es un consumo muy importante, entonces claramente la industria se ve afectada por el tipo de productos, porque además me parece que hoy la clasificación revela muchas verdades.

Todas estas líneas de productos que, tras una imagen de saludable porque están reducidas, porque están adicionadas, porque les coloca un determinado rótulo que incluye, por ejemplo, que son productos bio. Toda esta línea de productos que, lamentablemente, muchas veces la población asocia con productos más saludables, se ven atacados por esta clasificación también. Uruguay también comenzó la aplicación de etiquetado frontal, y tomando inicialmente el perfil de nutrientes de la OPS, con límites algo más flexibles. Y, por supuesto, la población se encuentra con la sorpresa que hay muchos productos que están reducidos en azúcares, en sodio, en grasa, pero que, sin embargo, tienen el etiquetado. Entonces, creo que esta es una clasificación que revela muchas verdades, que quita muchos mitos. Estos productos que, a través de la publicidad, a través de un marketing muy agresivo, nos inducen a creer de verdad que son alimentos saludables, y hasta muchas veces necesarios para mantener una salud o un crecimiento y un desarrollo adecuado en el caso de los niños. El marketing es muy agresivo y los productos que se publicitan, donde la industria de alguna forma trata de guiarte, son productos ultraprocesados, son productos superfluos, cuyo consumo tiene que inducirse a través de la publicidad. No vemos publicidad de vegetales, de fruta, por lo menos en nuestro país, la leche, la fruta, las verduras, las carnes sin procesamiento, no se publicitan. El marketing y la inversión de la industria están destinados a hacer crecer esos otros productos, basados en pocas materias primas, seguramente de muy poco costo, donde la gran inversión está puesta en el marketing y en crear un valor agregado, en base a crear una imagen de ese producto, que no está vendiendo precisamente su valor nutricional, sino otras cuestiones que nada tienen que ver con la alimentación. Entonces, me parece que la industria, de alguna manera, ve en peligro sus franjas de ventas más preciosas y que han tenido el mayor crecimiento en los últimos años. Creo que también los técnicos, los profesionales vinculados con el desarrollo de productos se sienten de alguna forma, no quiero decir amenazados, pero no valoran adecuadamente el término ultraprocesados, porque se dedican al procesamiento de productos muchos de ellos. Entonces, indirectamente ven como que están siendo atacados, porque la palabra en sí misma, ellos asocian que tiene una connotación negativa sobre su trabajo. Creo que esto es una cuestión de poder encontrar puntos en común. Es una clasificación que todavía sigue siendo relativamente nueva en algunos sectores. Entonces, me parece que, por eso le digo que la debilidad está en la clasificación química; de alguna forma, se ha de poder acercar o encontrar un punto en el cual haya también un acercamiento con quienes trabajan en el desarrollo de productos, para que puedan integrar también esta clasificación.

Simón Sí, yo creo que uno de los problemas es que, claramente, estos productos tienen un impacto en salud. Entonces, ya se vuelve un tema donde hay una serie de derechos, que son de mayor peso en la toma de decisiones, y ese yo creo que es el quid de la situación. En muchas otras industrias, cuando se trata de regular en el país, pues tienen todo el derecho de defender sus posturas, y de proponer las formas que más les convengan de hacer negocio. Aquí, en México, lo que nos ha preocupado mucho es que este tipo de respuesta de la industria, o de los profesionales que se dedican a esta tecnología de alimentos, puesto que es básicamente una postura de defensa de su negocio, pero en contra de la salud, del derecho a la información, del derecho al no marketing. Entonces, sí se vuelve un problema, un conflicto, pero yo creo que es un conflicto donde, sin duda, la tendencia y lo que tiene que pasar para que salgamos de este problema, que, además, como han visto, se ha acentuado con esta epidemia de COVID-19 10, es que tenemos que cambiar el modelo. Yo creo que para eso ayuda mucho esta clasificación NOVA. 
Veo esta discusión aquí también, esta discusión de los tecnólogos de alimentos, donde dicen: "es que eso no quiere decir ultraprocesado; yo te demuestro que el aceite de oliva también tiene muchísimos procesos". Y, bueno, yo creo que es una discusión semántica, no importa cómo le llamemos, sabemos muy bien desde el lado de la salud pública y de la opinión pública, a qué nos referimos con este ultraprocesado, a qué efecto está teniendo esto en la salud, y no lo queremos. Eso yo creo que no tiene controversia. Puede haber controversias semánticas, en los términos que utilicemos, pero yo creo que está muy claro las acciones que tenemos que tomar como profesionales de la salud pública y lo que no tiene que ocurrir. Estos productos que se están consumiendo en estas cantidades tan altas en la población, pues tiene que ir cambiando. Aquí, México también es una joya como ejemplo, por las bajas tasas de lactancia materna exclusiva en edades tempranas de la vida, por la introducción muy temprana de bebidas azucaradas y, después, el reemplazo de alimentos tradicionales por alimentos como sopas instantáneas, alimentos enlatados, muy altos en estos ingredientes críticos, preocupa. El otro sueño que yo creo que tienen los tecnólogos en alimentos, pienso que no tiene mucho futuro ya, en esta nueva tendencia mundial por la salud, era el de: “ipor qué no? Entonces, dígannos qué necesitan y nosotros con tecnología se lo damos, ¿quieren algo dulce que no engorde? Nosotros inventamos un sabor dulce y se lo damos bien dulce, y que no engorde, porque no tiene calorías". No, no es un modelo que yo creo que tenga futuro, esto de estar reemplazando todo el azúcar por edulcorantes no calóricos, cuyos efectos desconocemos, pero sabemos que no son metabólicamente inertes. Esto de ponerle cafeína a las bebidas para que tengan algún efecto. No es algo donde vayamos encontrar muchos consensos con este tipo de industria. Ahora, sí veo una oportunidad para la industria, la industria se tiene que adaptar, y tiene que ir buscando los alimentos menos procesados, además de formas más sostenibles de almacenaje, embalaje, y que pueda comercializar para subsistir, pero definitivamente no, no es el modelo que hemos tenido en los últimos años.

Ximena Me gustaría agregar en el tema que él dijo que era una discusión semántica, y yo estoy de acuerdo con esto. Me parece que es un problema que tenemos entre los técnicos, no con la población. Esto es una discusión sólo técnica. Cuando nosotros hicimos la guía alimentaria, se realizó la validación de los mensajes. Fue un trabajo realizado por dos grupos de investigación de la Universidad de la República, de Uruguay, y se aplicaron metodologías cuantitativas y cualitativas 11. La cuantitativa se hizo sobre todo en encuestas online, porque era un método rápido y barato, de bajo costo, y Uruguay tiene una muy alta cobertura de conectividad. Nosotros manejamos esas encuestas, en bases de datos bastante grandes con trabajadores activos, había toda clase de trabajadores, y cuándo se les pedía a las personas, tanto en la encuesta online, como en los grupos focales, que también se hicieron, la gente en general no sabe definir lo que es un ultraprocesado, es verdad, pero cuando uno le pide que coloque alimentos dentro de ese grupo, no hay mayores errores, o sea, realmente no hay un malentendido ahí, en relación a cuáles son los alimentos que corresponden, por supuesto, no es perfecta esa clasificación, pero sí es bastante cercana, acertada, o sea, me parece que la gente entiende bien a qué nos referimos, cuando hablamos de ultraprocesados 11.

João Me gustaría preguntar sobre esta tendencia a la reformulación. Nosotros vemos en algunos países, en la industria, incluso, sectores reformulando productos para que tengan pocos ingredientes, para que no tengan aditivos, no tengan aditivos artificiales, pero son todavía pocos productos. Y caros, muchas veces. Entonces, ¿̇cómo ven ustedes esta tendencia a la reformulación? De hecho, ¿̇entro de 10-15 años tendremos productos más sanos o esto no es nada más que otra tendencia de apropiación de algo que viene de una demanda de la sociedad por productos más sanos?

Simón Yo creo que sí, lo están tomando en serio, o sea, yo he visto los reportes que hacen, porque ellos analizan las tendencias en el mundo. Entonces, cuando venía este mercado fitness y todo eso, bueno, pues ellos van para allá. Ellos ven esta tendencia y, obviamente, están tratando de encontrarle la fórmula. Seguro que los que primero encuentren productos que cumplan con lo que esta tendencia busca, pues van a tener beneficios. Veo, por ejemplo, que en la crítica se buscan mucho las excepciones. Todos los sistemas tienen sus defectos, o sea no hay sistemas perfectos para clasificar y una de las cosas que pasa es que buscan como el caso más extremo, el que puede parecer más absurdo y ese lo usan como estandarte para criticar el sistema, ¿no? Entonces, yo creo que esa es una de las cosas que uno tiene que identificar, ¿cómo poder, en casos excepcionales, por un lado, defender el sistema, y, por otro lado, no castigar productos que son excepcionales? Por ejemplo, en México se discute mucho que se quiere un mayor consumo de oleaginosas, porque realmente es muy bajo el consumo, y de 
repente el sistema, como lo diseñamos, si hubiera unas almendras y estuvieran ahumadas, se podrían calificar, si se califica de manera integral el producto, como altas en grasa. Cosa que no les pasaría a las almendras naturales, porque las naturales no son evaluadas, porque es un producto básico. Este tipo de excepciones son lo que complica, y lo que hace susceptible de crítica el sistema. Una de las cosas que hicimos en esta primera etapa es no evaluar así las almendras ahumadas, no saldrían altas en grasa, no se valorarían en su totalidad. Esa es una de las cosas que pienso, y si la industria pasa de 3 sellos a 2 sellos, o a un sello, es decir, si en la reformulación van mejorando el perfil, eso les va a beneficiar, porque el mensaje en salud pública que uno debe dar es algo así como el de Chile: elige productos, sin sellos o con menos sellos, o si no tienen sellos mejor. Yo creo que, de todas maneras, aunque al final logremos que los productos tengan menos ingredientes críticos, el futuro, no digamos el inmediato, pero el medio plazo va a ser todavía más ultraprocesado. No pensando tanto, si tienen mucha sal o azúcar o grasa, es ultraprocesado, no es tan conveniente.

Ximena Nosotros justamente por un poco estas críticas y el proceso de validación, cuando colocamos el mensaje en la guía alimentaria, no hablamos de ultraprocesados en general, sino ultraprocesados con excesiva cantidad de grasas, azúcar y sal, porque fue una forma de trascender algunas críticas. En un momento donde realmente fue bastante dura la batalla por colocar el término en la guía alimentaria, hubo bastante presión, esa fue una fórmula como bastante salomónica de poder trascender, y a su vez buscar también alguna manera de identificarlos. Tenemos que avanzar hacia un sistema de etiquetado frontal que nos pueda decir cuáles son los productos con exceso, no digo que ese fue el origen único de la política de etiquetado frontal, pero, bueno, ayudó también. Nosotros estamos viendo que las reformulaciones, por lo menos hasta ahora, están más enfocadas en evitar la colocación de los sellos que en mejorar más cualitativamente el producto.

João Simón, ¿cómo se ha dado entre ustedes la decisión de adoptar el perfil de nutrientes de OPS, así como el perfil para los sellos? y ¿cómo se ha hecho la comparación con otros perfiles de nutrientes? ¿Qué se ha descubierto en su aplicación práctica? ¿Qué es lo que tiene como puntos fuertes y puntos débiles?

Simón Cuando estuvimos en el proceso de evaluar un sistema de etiquetado frontal para México, que eso lo empezamos en el 2007, es decir, hace muchos años, incluso antes de que hubiera etiquetado de advertencia, ya estábamos buscando un sistema. Empezamos tratando un sello de estos que califican de manera positiva al 20\% de los productos en el mercado, de tipo Choices International. Entonces, es decir, los productos que tengan el mejor perfil van a quedar calificados muy bien y tendrán este sello, que de todas maneras la industria tampoco lo quiso. Ahora, juy!, les hubiera encantado. No lo cambiarían, pero en ese ínter, donde en México estuvimos explorando posibilidades, estuvimos evaluando el ambiente alimentario y una de las cosas que hicimos fue estar tomando fotografías de productos. Entonces, tenemos más de 18.000 productos en los últimos dos o tres años, recolectados de todos los tipos de productos ultraprocesados que hay en México, que son muchísimos, y eso nos ha permitido evaluar un poco los perfiles. Entonces, podemos evaluar un sistema hipotético de etiquetado y cómo calificaría los productos, etcétera. Por otro lado, una cosa muy buena que pasa en Latinoamérica es que estamos con las redes y con el internet, y con todo esto hay una comunicación muy estrecha, yo diría que es una comunicación en tiempo real entre los países latinoamericanos, incluido Brasil, Argentina, Chile, Uruguay, México, Colombia, Perú, todos estos países están muy estrechamente comunicados y la Academia no decepciona, puesto que nosotros estamos muy comunicados con nuestros pares académicos. Entonces, hemos visto cómo se ha desarrollado todo este paradigma en Brasil, lo hemos utilizado, hemos utilizado las clasificaciones, fuimos de los primeros países en aplicarla en México, y ver cómo funcionaba. Con el etiquetado de advertencia, lo que hicimos es formar un comité internacional rápido, así por internet, trajimos a los expertos que tenían alguna experiencia en implementación de etiquetado frontal, entonces vinieron de Perú, de Ecuador, de Uruguay, de Chile y nos platicaron todos los problemas, de Brasil también vinieron, y este comité nos platicó todas sus experiencias, los problemas a los que se enfrentaron, lo que nos iba a decir la industria, nos lo dijeron antes que la industria, miren esto les van a decir, esto es muy difícil de defender. Con toda esa información, nosotros pudimos preparar una propuesta muy sólida, estábamos muy listos para defenderla, teníamos el material. 
Otra cosa que hicimos fue correr los modelos, y comparando el perfil por ejemplo de Chile, con el perfil de OPS, con el perfil con distintos sistemas de clasificación. Vimos, por ejemplo, que, con el perfil de Chile, pues sí queda mucho mejor que para calificar alimentos problemáticos que lo que nosotros teníamos en México, pero sin duda, si aplicáramos el perfil de OPS, quedaba mucho mejor. Es decir, identificaba alimentos problemáticos. Un ejemplo es que en Chile para ponerle el sello de alto en calorías a un producto hay un punto de corte, que es indistinto en líquidos y en sólidos. Entonces, el refresco, por ejemplo, una Coca-Cola solo tiene un sello, que es alto en azúcar, pero un yogur podía tener alto en azúcar, alto en calorías e incluso podría decir alto en grasa. Tres sellos, un yogur líquido y un sello Coca-Cola. Entonces alguien podría pensar que una Coca-Cola es mejor, porque tiene menos sellos, según la recomendación de política de si tiene menos sellos es mejor. Bueno, en México lo que hicimos fue, por un lado, cambiar el punto de corte para líquidos, entonces, los refrescos o cualquier bebida, si llega a tener el equivalente a más de $5 \mathrm{~g}$ por una taza, es decir, $5 \mathrm{~g}$ x $250 \mathrm{~mL}$ o 2,5 por $100 \mathrm{~mL}, 2 \mathrm{~g}$ por $100 \mathrm{~mL}$, quedaría como alto en calorías, entonces una Coca-Cola tendría dos sellos y además tendría una leyenda de que contiene cafeína, y que no, ese producto no se recomienda en niños. Entonces eso fue parte de estar modelando y viendo cómo quedaban los ingredientes en esta gran base de datos que teníamos de más de 18.000 productos. Otra cosa que nos permitió esta base, es que cuando la industria decía no, es que ustedes están haciendo un sistema con el que todos nuestros productos van a quedar llenos de sellos, y así lo decían en los medios y en el periódico, entonces rapidísimo les decíamos no, nosotros tenemos aquí una base de 18.000 productos, y ¿qué creen? $13 \%$ de los productos, o $14 \%$ de los productos, no van a tener ningún sello; hay como un $14 \%$ que van a tener un sello; hay tantos que van a tener 2 y 3 sellos, y casi ninguno va a tener cuatro sellos. Además, como nuestro sistema tiene más sellos, tiene sellos para grasas trans, para edulcorantes, para cafeína; es que el sistema mexicano tiene 8 sellos. Ningún sistema en el mundo tiene 8 sellos, pero bueno, les demostramos con esto, con esta evidencia, pues que la mayor parte de los productos no pasarían de cuatro sellos. Eso ayudó también mucho.

Ximena [aquí se retiró un párrafo que perdió parte del sentido, debido a cambios coyunturales]. A diferencia de México, nuestra propuesta de etiquetado frontal, la que está vigente hasta el momento, si bien hay un decreto de prórroga de aplicación del decreto, incluye 4 sellos que son: azúcares, grasas, grasas saturadas y sodio. Nosotros no tenemos advertencia para edulcorantes. Entonces un posible riesgo es la sustitución de azúcar por edulcorantes, claramente. Por eso, si bien estamos en un párate desde la elaboración del decreto, siempre pensamos que la campaña no debería estar dirigida solamente a consumir alimentos con menos sellos, sino que de alguna manera tenía que ir acompañado de un mensaje. Así, pensamos la campaña que hicimos durante el año pasado, para valorizar la comida casera y los alimentos naturales, porque si no, corremos un riesgo de terminar consumiendo alimentos reformulados, que pueden tener una ventaja, si uno los compara con el alimento original, pero que sigue siendo un producto ultraprocesado, y lo que nosotros necesitamos impulsar en la población es que base su alimentación en alimentos naturales, y que este tipo de productos sean la excepción y no la regla. Entonces, por ahí han estado un poco centrados los esfuerzos, que suponen un gran desafío, en poder, por un lado, instaurar y defender el etiquetado frontal, argumentando que esto va a tener obviamente un beneficio para la población, en términos sobre todo de información y poder tomar decisiones informadas, pero, por otro, seguir defendiendo la vuelta a las prácticas culinarias más tradicionales, defender los alimentos naturales, la producción local, y, el comercio de cercanía y las comidas caseras.

Hay que trabajar las dos cosas al mismo tiempo, porque si no el mensaje termina siendo: consuma ultraprocesados, pero con menos sellos. También entiendo que nuestro etiquetado no es, no tiene tantas características como cuentan en otros modelos, donde se introducen otros sellos que nosotros no tenemos. Nosotros quisimos introducir una advertencia, no con la misma connotación, y el mismo formato de los excesos de nutrientes críticos para los edulcorantes. Era simplemente colocar en letra más clara, en la parte frontal del envase, la presencia de edulcorantes, pero no hubo consenso, sobre todo, porque no se comprendía cuál era la ventaja de esto a nivel del Mercosur. Sobre todo, porque nosotros, obviamente, pertenecemos a esa región. 
João En el caso de la guía, ¿cómo otros sectores de gobierno, Agricultura, Desarrollo Social, Economía han dialogado con el documento? ¿Se han creado otras políticas públicas desde el documento?

Ximena Tuvimos un grupo de consenso bastante amplio, donde se incluye, si uno recorre la guía, muchos sectores. Participó, por supuesto, el Ministerio de Desarrollo Social, el Ministerio de Educación, Ganadería, Agricultura y Pesca y una serie de instituciones gubernamentales relacionados con el tema, la academia y la sociedad civil. De alguna manera, intentamos, y por supuesto que es un gran desafío, trascender lo que es la guía alimentaria como una herramienta puramente educativa, sino que se adopte como un lineamiento de política pública. Y en ese camino estamos, por supuesto, tenemos un decreto también que conforma una comisión para abordar lo que es el sobrepeso y la obesidad, para desarrollar políticas públicas que aborden este problema y una de las líneas de trabajo es la implementación de los lineamientos de la guía alimentaria en otras políticas públicas. Y, claramente, uno de los grandes desafíos es crear entornos educativos saludables. En esa línea, una vez aplicado el decreto, debemos reformular lo que es la lista de alimentos recomendados para la venta en los centros educativos. Tenemos una ley, que es la Ley $n^{\circ} 19.140$, que le da la competencia al Ministerio de Salud de elaborar el listado de alimentos recomendados, donde nosotros tenemos un grupo 1, que son los naturales, un grupo 2, que son las preparaciones más caseras, y el 3, que son los productos envasados con altos contenidos de nutrientes críticos. A partir del etiquetado íbamos a reformular ese listado que ya tiene, introducida la mirada de la clasificación NOVA, pero que se podía profundizar más a partir de la introducción del etiquetado. De hecho, tenemos publicaciones que están disponibles en la página web del Ministerio, donde ya se introduce la exclusión de ese listado de los productos ultraprocesados con alto contenido de grasas, azúcar y sodio.

En esta coyuntura del COVID-19, el Ministerio de Desarrollo Social ha realizado algunos protocolos, en términos de lo que son donaciones. Esta también es una oportunidad, de alguna forma, para que la industria de alimentos canalice productos, que después no queremos que se introduzcan en la familia, y luego se transformen en un hábito. Por ello, se establece un protocolo para evaluar las donaciones y, en el caso de los productos ultraprocesados, se analiza por un comité la pertinencia de aceptar esa donación. En el caso de que se acepte, se analiza ¿cuál sería el destino de esa donación? Para no interferir, justamente, con la alimentación que se quiere promover. 


\section{Colaboradores}

J. Peres participó en la redacción del texto y revisión de la versión final que se publicará. S. Barquera y X. Moratorio revisaron la versión final que se publicará.

\section{Informaciones adicionales}

ORCID: João Peres (0000-0002-4767-2811); Simón Barquera (0000-0003-1854-4615); Ximena Moratorio (0000-0002-4403-9113).
1. Pan American Health Organization. Ultra-processed food and drink products in Latin America: Sales, sources, nutrient profiles, and policy implications. Washington DC: Pan American Health Organization; 2019.

2. Monteiro CA, Levy RB, Claro RM, Castro IRR, Cannon G. A new classification of foods based on the extent and purpose of their processing. Cad Saúde Pública 2010; 26:2039-49.

3. Barquera S, Hernandez-Barrera L, Tolentino ML, Espinosa J, Ng SW, Rivera JA, et al. Energy intake from beverages is increasing among Mexican adolescents and adults. J Nutr 2008; 138:2454-61.

4. Goberno de la Ciudad de México. Ley del Impuesto Especial sobre Producción y Servicios. Diario Oficial de la Federación 2013; 8 sep.

5. Secretaría de Economía. Norma Oficial Mexicana NOM-051-SCFI/SSAI-2010. Especificaciones generales de etiquetado para alimentos y bebidas no alcohólicas preenvasados. Diario Oficial de la Federación 2010; 18 feb.

6. Organização Pan-Americana da Saúde. Modelo de perfil nutricional da Organização Pan-Americana da Saúde. Washington DC: Organização Pan-Americana da Saúde; 2016.
7. Ministerio de Salud. Guía alimentaria para la población uruguaya: para una alimentación saludable, compartida y placentera. Montevideo: Ministerio de Salud; 2016.

8. Ministerio de Desarrollo Social. Protocolo para la evaluación de donaciones de alimentos, INDAMIDES. Montevideo: Ministerio de Desarrollo Social; 2020.

9. Organización Panamericana de la Salud. Alimentos y bebidas ultraprocesados en América Latina: ventas, fuentes, perfiles de nutrientes e implicaciones. Washington DC: Organización Panamericana de la Salud; 2019.

10. Denova-Gutiérrez E, Lopez-Gatell H, AlomiaZegarra J, López-Ridaura R, Zaragoza-Jimenez $\mathrm{C}$, Dyer-Leal D, et al. The association between obesity, type 2 diabetes, and hypertension with severe COVID-19 on admission among Mexicans. Obesity (Silver Spring) 2020; [Epub ahead of print].

11. Ares G, Vidal L, Allegue G, Giménez A, Bandeira E, Moratorio X, et al. Consumers' conceptualization of ultra-processed foods. Appetite 2016; 105:611-7.

Recibido el 03/Nov/2020

Versión final presentada el 07/May/2021

Aprobado el 31/May/2021 